
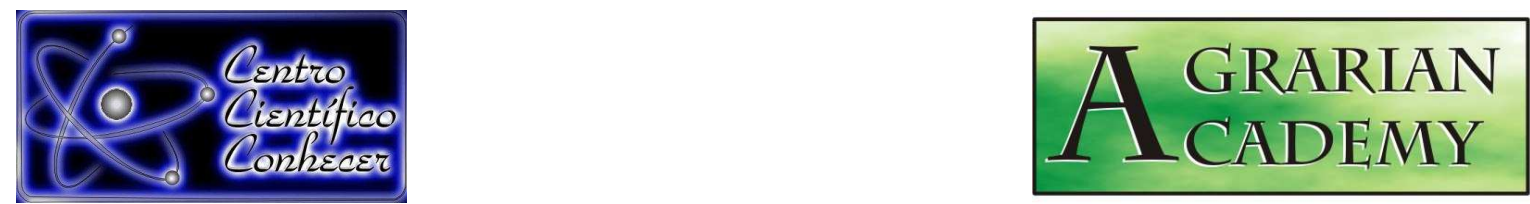

\title{
DIFERENTES GRADES DE AMOSTRAGEM PARA AVALIAÇÃO DE ATRIBUTOS QUÍMICOS DO SOLO NA DISTRIBUIÇÃO ESPACIAL EM ÁREAS DE CANA-DE- AÇÚCAR
}

Anderson Antonio da Silva ${ }^{1}$, Marcos Rafael Nanni ${ }^{2}$, Carlos Antonio da Silva Junior ${ }^{1}$, Franciele Romagnoli ${ }^{1}$, Marcelo Luiz Chicati $^{3}$, Aline de Carvalho Gasparotto ${ }^{1}$

${ }^{1}$ Universidade Estadual de Maringá-UEM, Pós-Graduação em Agronomia, Avenida Colombo, 5790, Jardim Universitário, CEP: 87020-900, Maringá, PR. E-mail:

Anderson_agrouem@hotmail.com.

${ }^{2}$ Universidade Estadual de Maringá-UEM, Departamento de Agronomia, Avenida Colombo, 5790, Jardim Universitário, CEP: 87020-900, Maringá, PR.

${ }^{3}$ Universidade Estadual de Maringá-UEM, Departamento de Engenharia Agrícola, Campus do Arenito. Rodovia PR 482, Km 45, CEP: 87820-000, Cidade Gaúcha, PR.

Recebido em: 03/01/2014 - Aprovado em: 04/04/2014 - Publicado em: 12/04/2014

\begin{abstract}
RESUMO
O objetivo deste trabalho foi quantificar e avaliar a distribuição espacial de atributos químicos dos solos em diferentes grades de amostragem, em uma área de estudo localizada no município de Bom Sucesso, no estado do Paraná, por meio de análises estatísticas descritivas e geoestatística. Foi estabelecida uma grade de amostragem com o tamanho de aresta de $50 \times 50 \mathrm{~m}$, compreendendo quatro amostras por hectare, por meio de sistema GPS. Cada ponto de coleta foi locado a campo de onde se extraiu as amostras de solo do horizonte superficial na profundidade de 00-0,20m, numa área de 51,15ha totalizando 203 pontos de amostragem. Das amostras foram determinados os valores de $\mathrm{pH}$ e Carbono. Grades com menores intensidades amostrais foram estabelecidas a partir da grade inicial, retirando-se amostras aleatoriamente, gerandose grades de: $70,8 \times 70,8 \mathrm{~m}$ ( 2 amostras/ ha), $100 \times 100 \mathrm{~m}$ ( 1 amostra/ha),141,4 $\mathrm{x}$ $141,4 \mathrm{~m}$ (1 amostra/2ha),173,3 x 173,3m (1 amostra/3ha) e $200 \times 200 \mathrm{~m}$ (1 amostra/4ha). Os dados obtidos de cada um dos atributos químicos do solo, nas diferentes grades amostrais foram submetidos a análise estatística descritiva e estudo geoestatístico, construindo semivariogramas experimentais para compreender sua estrutura de dependência espacial. Os atributos $\mathrm{pH}$ e Carbono apresentaram dependência espacial em pelo menos uma das grades amostrais. Os alcances dos atributos químicos $\mathrm{pH}$, Carbono variaram de 240 à $800 \mathrm{~m}$. Os semivariogramas do atributo $\mathrm{pH}$ apresentaram estrutura de dependência espacial fraca a moderada, já o semivariograma do Carbono apresentaram dependência espacial moderada.
\end{abstract}

PALAVRAS-CHAVES: Distribuição espacial de atributos, dependência espacial, estatística descritiva, geoestatística, grade de amostragem. 


\title{
DIFFERENT GRIDS OF SAMPLING FOR ASSESSMENT OF CHEMICAL ATTRIBUTES OF SOIL IN SPATIAL DISTRIBUTION IN AREAS OF SUGARCANE
}

\begin{abstract}
The objective of this study were to quantify and assess the spatial distribution of chemical properties of soils at different sampling grids, in a study area located in the municipality of Bom Sucesso, in the state of Paraná, through descriptive statistical analysis and geostatistics. A grid sampling edge to size $50 \times 50 \mathrm{~m}$ was set comprising four samples per hectare using GPS. Each collection point was leased the field from which extracted soil samples from the surface horizon at a depth of 00 to $0.20 \mathrm{~m}$, in an area of 51.15 ha total of 203 sampling points. The samples were determined $\mathrm{pH}$ and Carbon. Grids with smaller sample intensities were established from the initial grid, removing samples randomly, generating grids: $70.8 \times 70.8 \mathrm{~m}$ ( 2 samples/ha, $100 \mathrm{x}$ $100 \mathrm{~m}$ ( 1 sample/ha ), $141,4 \times 141.4 \mathrm{~m}$ (1 sample/2ha), 173.3 x $173.3 \mathrm{~m}$ (1 sample/3ha) and $200 \times 200 \mathrm{~m}$ ( 1 sample/4ha). The data obtained for each soil chemical properties in different sample grids were subjected to descriptive statistical analysis and geostatistical study, building experimental semivariogram to understand the structure of spatial dependence. The $\mathrm{pH}$ and Carbon attributes spatially dependent in at least one of the sampling grids. The ranges of chemical properties $\mathrm{pH}$, carbon ranged from 240 to $800 \mathrm{~m}$. The semivariogram $\mathrm{pH}$ attribute structure showed weak to moderate spatial dependence, since the semivariogram Carbon showed moderate spatial dependence.
\end{abstract}

KEYWORDS: Grid sampling, spatial attributes, spatial dependence, descriptive statistics, geostatistics.

\section{INTRODUÇÃO}

A agricultura brasileira, nas últimas décadas, passou por constantes mudanças e avanços tecnológicos, rápida automação pela introdução de máquinas e implementos agrícolas, com auxílio de controles computacionais e tecnologia de posicionamento global (GPS), melhoramento genético de plantas, novos princípios ativos e posicionamento de defensivos agrícolas, tecnologia de liberação controlada em fertilizantes, em busca do aumento da produção e redução de impactos negativos ao ambiente (SILVA, 2013).

Embora vários trabalhos tenham sido realizados na área de variabilidade espacial abrangendo, principalmente, a relação solo-planta, os mesmos não têm alcançado a devida difusão entre técnicos e engenheiros na extensão rural.

Informações sobre variáveis ligadas à produção agrícola são bastante divulgadas, mas pouco conhecidas quanto ao seu comportamento espacial. Algumas caracterizações didáticas como "ambiente de produção", são constantemente preconizadas no setor agrícola, porém bastante subjetiva a quem estabelece os critérios, avaliações e componentes de produção (SILVA, 2013).

$\mathrm{Na}$ busca de tentar compreender e caracterizar melhor a espacialização das variáveis de produção, estabelecendo melhores parâmetros e critérios para classificar os "ambientes de produção", projetos e pesquisas são necessários para que possam contribuir e auxiliar nas práticas e decisões agronômicas.

O estudo mais aprofundado da variabilidade espacial dos atributos físicos, químicos e biológicos do solo faz-se necessário, uma vez que as práticas agrícolas de 
manejo são dependentes desta variabilidade, com impacto direto na produção agrícola e ambiente. Acredita-se ser a forma mais elaborada para entender as variáveis de produção ligadas ao sistema solo-planta.

O conhecimento destas variações é imprescindível para o levantamento e classificação dos ambientes com potencial produtivo avaliação da sua fertilidade, desenvolvimento de sistemas adequados de amostragens, planejamento experimental e definições de práticas apropriadas de manejo e recuperação de áreas de produção agrícola.

Sendo assim, neste trabalho buscou-se estudar e avaliar os atributos químicos $\mathrm{pH}$ e Carbono no solo em função de sua distribuição e comportamento espacial, visando fornecer subsídios ao manejo localizado em área de produção de cana de açúcar.

\section{MATERIAL E MÉTODOS}

A área escolhida para a realização dos estudos está localizada no município de Bom Sucesso, ao noroeste do Estado do Paraná, delimitada pelas coordenadas UTM e projeção SAD 69, com base no fuso 22 sul e meridiano 51o W.G. $420291 \mathrm{~m}$ - 421699 m e 7382200 m - 7383393 m do Equador. O município de Bom Sucesso (Figura 1), fundado em 1954, possui área total de $323 \mathrm{~km} 2$, e altitude média de $580 \mathrm{~m}$ tendo como referência altimétrica o Marégrafo de Imbituba.

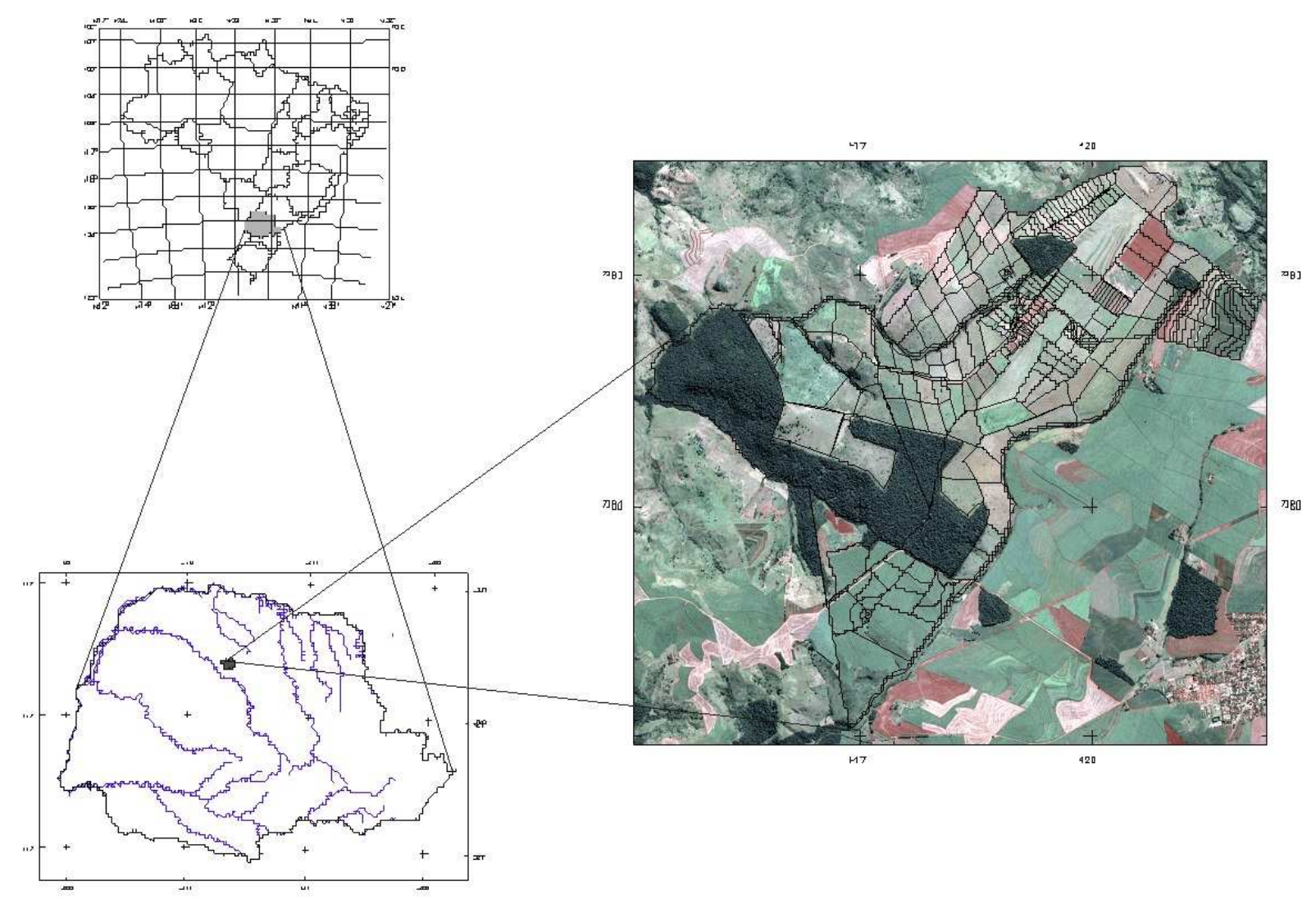

FIGURA 1. Localização do Município de Bom Sucesso e da área de estudo 
Para realização das atividades de laboratório/escritório foram utilizados os seguintes programas computacionais: Excel, para montagem das planilhas de dados; o Sistema para Processamento de Informações Georreferenciadas (SPRING versão $5.2 .2,2012)$ para elaboração do banco de dados e toda a manipulação de informações georreferenciadas; SMS (Spatial Management System) para produção das grades regulares; AutoCad para trabalhar os vetores (linhas) dos mapas ; Surfer (versão 8.01, 2002) para confecção de mapas 3D e software VARIOWIN2.2 (PANNATIER, 1996), para realização das análises de dependência espacial e STATISTICA 8 (Statsoft) para análise de estatística descritiva e o modulo geoestatístico/validação do SPRING 5.2.2 (INPE) para interpolação dos atributos do solo pelo interpolador krigeagem.

O trabalho iniciou-se pela preparação do mapa em sistema CAD no software AutoCAD e trabalhado no sistema de informações cartográficas para inserir o mesmo num banco de dados. Este mapa foi readequado dentro do Sistema de Processamento de Informações Geocodificadas - SPRING desenvolvido pelo INPE e exportado para o software SMS para a produção de grades regulares de amostragem e re-importado novamente para SPRING. Segundo CÂMARA et al. (1998), o SPRING é um sistema de geoprocessamento de última geração idealizado por programação orientada-a-objeto, com múltiplas funções e algoritmos para processamento de banco de dados georreferenciados.

Para realização da coleta das amostras de solo, foi estabelecida uma grade regular (Figura 3) de amostragem, distante de 50 em 50 metros como grade base (referência), compreendendo quatro amostras por hectare numa área de 51,14 ha.

Outras grades foram estabelecidas a partir desta diminuindo a quantidade de pontos amostrais (NANNI et al., 2011): 70,8 x 70,8m compreendendo duas amostras por hectare; $100 \times 100 \mathrm{~m}$ compreendendo uma amostra por hectare; $141,4 \times 141,4 \mathrm{~m}$ compreendendo uma amostra a cada dois hectares; 173,3 x 173,3m compreendendo uma amostra a cada três hectares; 200 x 200 m compreendendo uma amostra a cada quatro hectares (Figura 2). 


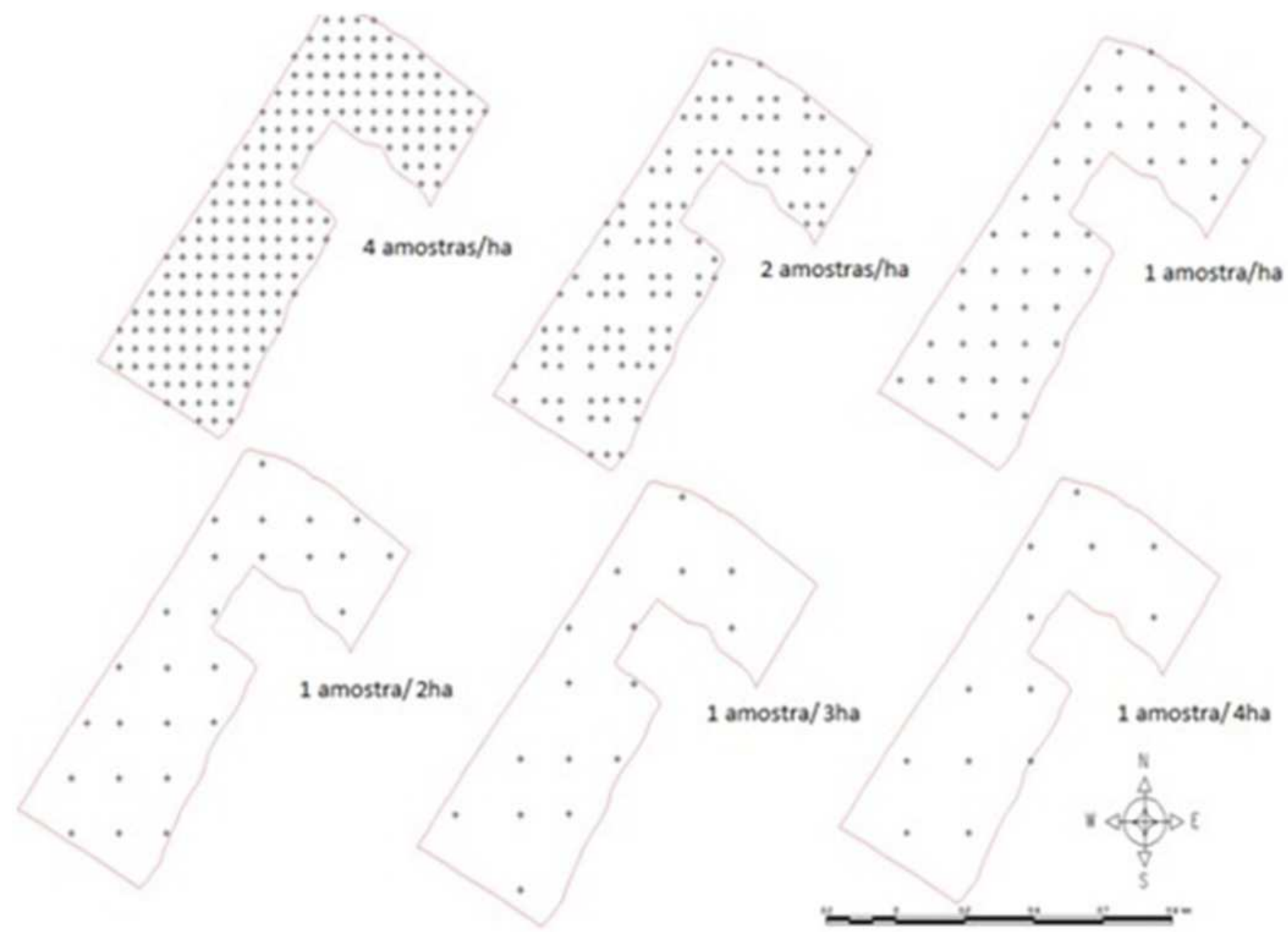

FIGURA 2. Grades regulares de amostragem.

A amostragem foi realizada por meio de tradagens nas profundidades de $0-0,20$ $\mathrm{m}$ no horizonte superficial para a grade de $50 \times 50 \mathrm{~m}$ uma amostra por ponto sem subamostra. A profundidade de $0-0,20 \mathrm{~m}$ foi escolhida para análise por ter sido considerada a profundidade efetiva mais explorada pelo sistema radicular da cultura da cana-de-açúcar, além de representar o horizonte diagnóstico de correção/fertilidade.

Para as coletas de amostras de solo, os pontos foram localizados pelo sistema GPS, facilitando o processo de prospecção, pois forneceu a coordenada de todos os locais dos pontos a serem visitados, diminuindo a possibilidade de erros e permitindo a possibilidade de retornar ao mesmo ponto para novas coletas de confirmação.

As amostras foram acondicionadas em sacos plásticos, identificadas e conduzidas ao Laboratório Agronômico Rural de Maringá, para análise química macro nutrientes.

Os elementos químicos analisados foram: carbono (C), potencial hidrogeniônico $(\mathrm{pH})$, utilizando-se os métodos desenvolvidos pela EMBRAPA (1997).

Com a finalidade de analisar e comparar a distribuição espacial dos atributos: $\mathrm{pH}$ e Carbono, encontrar qual a melhor grade regular de amostragem (50; 70,8; 100; 141,$4 ; 173,3$ e 200m) dentre as estabelecidas que melhor caracteriza a distribuição espacial do atributo no solo e que retrata com fidelidade as condições locais.

Para cada atributo químico do solo estudado realizou-se análise estatística descritiva e a comparação de média pelo teste de Tukey ao nível de $5 \%$ de 
significância, com auxilio do software Statistica. Na estatística descritiva calculou-se a média, mediana, mínimo, máximo a variância, a assimetria, a curtose, o coeficiente de variação, desvio padrão e o tipo de distribuição. Utilizou-se o teste de Lilliefors (PValor) a 5\% de probabilidade para testar a hipótese de normalidade.

$\mathrm{O} \mathrm{pH}$ grade de 50,70,8,141,4m foram transformados distribuição log normal para atender a normalidade. Esse processo foi de extrema importância para posteriormente fazer uma análise espacial do conjunto de dados para o ajuste de seus respectivos semivariogramas.

Considerou-se, neste trabalho, que os dados atendem à condição de estacionaridade intrínseca que ocorre quando a esperança $(E)$ de $Z(x i)$ é constante, $\mu(x i)=\mu$ e a variância (VAR) do incremento entre $Z(x i)$ e $Z(x i+h)$ é finita e independente da posição no espaço, dependendo apenas da distância de separação $h$, já que os dados são simétricos em relação à normalidade (GONÇALVES, 1997; VIEIRA, 2000). Este nível de estacionaridade dos dados é suficiente para a aplicação da geoestatística.

Todos os conjuntos de dados nas diferentes grades regulares de amostragem da área de estudo foram submetidos a análise geoestatística na construção e interpretação do semivariograma experimental no software VARIOWIN 2.2 (PANNATIER, 1996) obtendo o ajuste do modelo matemático para explicar a estrutura de variância dos dados, Para tal, foi utilizado o semivariograma clássico de Matheron, que é dado pela Equação 1.

$$
\gamma(h)=\frac{1 \sum_{i=1}^{n}\left[Z_{i}\left(x_{i}\right)-Z_{i}\left(x_{i}+h\right)\right]\left[Z_{j}\left(x_{i}\right)-Z_{j}\left(x_{i}+h\right)\right]}{2 n}
$$

Após a geração dos semivariogramas experimental foram testados os modelos matemáticos teóricos, (a) esférico, (b) exponencial e (c) gaussiano, mostrados abaixo respectivamente, que de acordo com MCBRATNEY \& WEBSTER (1983) estão entre os ditos autorizados.

a) Esférico: $\quad \mathrm{Y}(\mathrm{h})=\mathrm{C} 0+\mathrm{C}[1,5(\mathrm{~h} / \mathrm{A})-0,5(\mathrm{~h} / \mathrm{A}) 3]$

b) Exponencial: $\quad \mathrm{Y}(\mathrm{h})=\mathrm{C} 0+\mathrm{C}[1-\exp (-\mathrm{h} / \mathrm{A})]$

c) Gaussiano: $\quad \mathrm{V}(\mathrm{h})=\mathrm{CO}+\mathrm{C}[1-\exp (-\mathrm{h} / \mathrm{A}) 2]$

Em que Co é o efeito pepita ou "Nugget", que é o valor de $\mathrm{y}(\mathrm{h})$ quando $\mathrm{h}=0$; $\mathrm{Co}+\mathrm{C} 1$ = patamar ou "Sill" é o valor de $\mathrm{Y}(\mathrm{h})$ quando a variância se estabiliza; $\mathrm{C}$ é a variância estrutural ou a diferença entre o efeito pepita e o patamar; e "a" é o alcance ou "Range", considerando a distância até onde o patamar se estabiliza e representa a amplitude da dependência espacial a partir da qual a variável se distribui ao acaso (GREGO \& VIEIRA, 2005).

Para descrever o comportamento e ajuste de modelo de semivariograma para cada atributo nas diferentes grades regulares, A "indicação da qualidade do ajuste" (indicative goodness of fit/IGF) é fornecida pela Equação 2. 


$$
I G F=\frac{1}{N} \sum_{k-1}^{N} \sum_{i=0}^{n(k)} \frac{P(i)}{\sum_{j-0}^{n(k)} P(j)} \bullet \frac{D(k)}{d(i)} \bullet\left[\frac{\gamma(i)-\gamma *(i)}{\delta^{2}}\right]^{2}
$$

Onde:

$\mathrm{N}$ = número de variogramas direcionais

$\mathrm{n}(\mathrm{k})=$ numero de passos (lags) relativo ao variograma $\mathrm{k}$

$D(k)=$ distância máxima relativa ao variograma $k$

$\mathrm{P}(\mathrm{i})=$ número de pares para o passo $\mathrm{i}$ do variograma $\mathrm{k}$

$\mathrm{d}(\mathrm{i})=$ distância média dos pares para o passo i do variograma $\mathrm{k}$

$\mathrm{Y}(\mathrm{i})=$ medida experimental da continuidade espacial para o passo $\mathrm{i}$

$\mathrm{Y}^{\star}(\mathrm{i})=$ medida modelada da continuidade espacial para $\mathrm{d}(\mathrm{i})$

$\sigma 2=($ co)variância dos dados para o variograma (cruzado).

O IGF é um número adimensional e valores quanto mais próximos a zero, melhor o ajuste indicado. A partir dos modelos obtidos, para os semivariogramas ajustados para cada atributo químico em suas respectivas grades de amostragem estabelecidas da área de estudo, estimaram-se os valores das variáveis químicas em locais não amostrados por meio do método de interpolação geoestatístico de Krigagem que é o melhor estimador linear não tendencioso (WEBSTER \& OLIVER,1990). Deste modo, com os valores estimados foi possível construir mapas de isolinhas que expressam a variabilidade das variáveis avaliadas na área em estudo.

\section{RESULTADOS E DISCUSSÕES}

Para melhor compreensão da distribuição dos atributos nas diferentes grades de amostragem avaliados na área de estudo, foram estabelecidos os valores da média aritmética dos atributos para cada grade, como demonstrado na Tabela 1.

TABELA 1. Médias aritméticas e coeficiente de variação (CV) dos atributos dos solos obtidos a partir das amostras escolhidas para as diferentes grades.

\begin{tabular}{|c|c|c|c|c|c|c|c|}
\hline Atributo & \multicolumn{6}{|c|}{ Grade } & CV \\
\hline & $50 \mathrm{~m}$ & $70,8 \mathrm{~m}$ & $100 m$ & $141,4 \mathrm{~m}$ & $173,3 m$ & $200 \mathrm{~m}$ & $(\%)$ \\
\hline $\mathrm{pH}$ & 5,2 & 5,3 & 5,2 & 5,2 & 5,3 & 5,2 & 0,78 \\
\hline Carbono & 22,1 & 21,5 & 21,6 & 22,6 & 24,0 & 23,5 & 4,63 \\
\hline
\end{tabular}

*Unidades: Carbono $\left(\mathrm{g} \cdot \mathrm{kg}^{-1}\right)$

De acordo com a EMBRAPA (2010), os valores médios dos atributos químicos do solo na camada de 0 - 0,20 m (Tabela 1), permitiram classificar a sua fertilidade como elevada para pH e Carbono, uma vez que o mesmo foi corrigido (Calagem).

Segundo VANNI (1998), se o coeficiente de variação for menor que $35 \%$, a série de dados pode ser considerada homogênea e a média pode apresentar significância, podendo ser utilizada como representativa da série de onde foi obtida. 
Para todas as grades regulares os CV's apresentaram-se com valores baixos sendo, provavelmente devido ao mesmo manejo de solos e tratos culturais (Adubação) estabelecidos para área.

Os resultados da análise descritiva dos atributos químicos do solo são apresentados nas Tabelas 2 e 3 separadamente. A aplicação do teste de normalidade ao conjunto de dados revela que os atributos: $\mathrm{pH}$ (com exceção das grades $50 \mathrm{~m}$, $70,8 \mathrm{~m}$ e $141,4 \mathrm{~m}$ ) e carbono (com exceção da grade de $70,8 \mathrm{~m}$ ) apresentam distribuição normal.

Analisando-se os resultados obtidos na estatística descritiva (Tabela 2), observa-se que os valores de $\mathrm{pH}$ demonstraram e mantiveram baixo $\mathrm{CV}$ em todas a grades regulares de amostragem $(<12 \%)$, segundo critérios propostos por WARRICK \& NIELSEN (1980).

Estes baixos valores, segundo DAVIS et al. (1995), podem ser decorrentes da função logarítmica utilizada na determinação do $\mathrm{pH}$ em $\mathrm{CaCl} 2$ apresentando, naturalmente, pequena variação, bem como os valores de desvio padrão (DP), com maior variação dos coeficientes de curtose e assimetria entre as grades analisadas.

Nas Grades de 50 e 70,8m não ocorreu distribuição normal para pH sendo seus valores ajustados na distribuição log normal (VIEIRA, 1997), como observado por MOREIRA et al. (2003).

TABELA 2. Estatística descritiva dos valores de $\mathrm{pH}$ para as diferentes grades de amostragem.

\begin{tabular}{lccccccccccc}
\hline Grade $^{1}(\mathrm{~m})$ & $\mathrm{N}^{2}$ & min & mediana & máx & méd & Curtose & Assim. & & $\sigma^{4}$ & $\mathrm{DP}^{5}$ & $\mathrm{P}^{6}$ \\
\hline 50 & 203 & 4,3 & 5,2 & 7,2 & 5,2 & 3,3 & 0,9 & 7,6 & 0,15 & 0,39 & $0,0^{*}$ \\
70,8 & 103 & 4,3 & 5,3 & 7,2 & 5,3 & 3,1 & 1,0 & 8,5 & 0,20 & 0,44 & $0,0^{*}$ \\
100 & 48 & 4,4 & 5,2 & 6,1 & 5,2 & $-0,3$ & 0,1 & 7,8 & 0,16 & 0,40 & 0,262 \\
141,4 & 26 & 4,5 & 5,2 & 6,2 & 5,2 & 1,2 & 0,4 & 6,7 & 0,12 & 0,34 & 0,049 \\
173,3 & 16 & 4,5 & 5,2 & 6,1 & 5,3 & $-0,6$ & 0,2 & 8,5 & 0,19 & 0,43 & 0,147 \\
200 & 13 & 4,7 & 5,1 & 5,9 & 5,2 & $-0,6$ & 0,6 & 7,0 & 0,12 & 0,35 & 0,055 \\
\hline
\end{tabular}

O Carbono (Tabela 3) também apresentou distribuição normal, CV e DP baixo nas diferentes grades, atendendo a normalidade com a aplicação log normal.

Como no caso anterior, a área em estudo recebe, decorrente da necessidade, corretivos de acidez (calcário dolomítico). Como o manejo da cultura é uniformizado, os teores e formas de carbono são, necessariamente, homogêneos decorrentes da queima da palha na colheita e os remanescentes da cultura da cana, como relatado por NANNI (2001). No entanto, diferentes resultados foram observados por CAVALCANTE et al. (2007). 
TABELA 3. Estatística descritiva dos valores de Carbono para as diferentes grades de amostragem.

\begin{tabular}{|c|c|c|c|c|c|c|c|c|c|c|c|}
\hline $\operatorname{Grade}^{1}(\mathrm{~m})$ & $\mathrm{N}^{2}$ & $\min$ & mediana & máx & méd & Curtose & Assim. & & $\sigma^{4}$ & & \\
\hline & & & & & & & & CV\% ${ }^{3}$ & & $\mathrm{DP}^{5}$ & $P^{6}$ \\
\hline 50 & 203 & 8,7 & 22,2 & 36,4 & 22,1 & $-0,17$ & 0,01 & 27,9 & 37,9 & 6,15 & 0,57 \\
\hline 70,8 & 103 & 8,9 & 22,2 & 36,4 & 21,5 & $-0,32$ & 0,00 & 28,7 & 37,7 & 6,14 & 0,69 \\
\hline 100 & 48 & 9,2 & 21,1 & 32,5 & 21,6 & $-0,17$ & $-0,12$ & 26,4 & 31,9 & 5,65 & 0,79 \\
\hline 141,4 & 26 & 9,2 & 22,2 & 35,2 & 22,6 & 1,29 & 0,01 & 23,4 & 27,0 & 5,20 & 0,47 \\
\hline 173,3 & 16 & 9,2 & 22,2 & 35,1 & 24,0 & 1,23 & $-0,31$ & 25,7 & 35,7 & 5,97 & 0,10 \\
\hline 200 & 13 & 9,6 & 23,9 & 36,1 & 23,5 & 0,74 & $-0,43$ & 29,9 & 45,8 & 6,77 & 0,10 \\
\hline
\end{tabular}

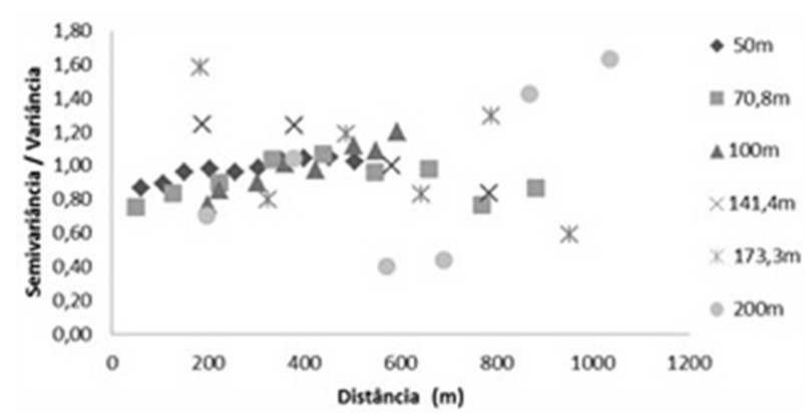

FIGURA 3. Semivariogramas de diferentes grades de amostragem para o atributo $\mathrm{pH}$.

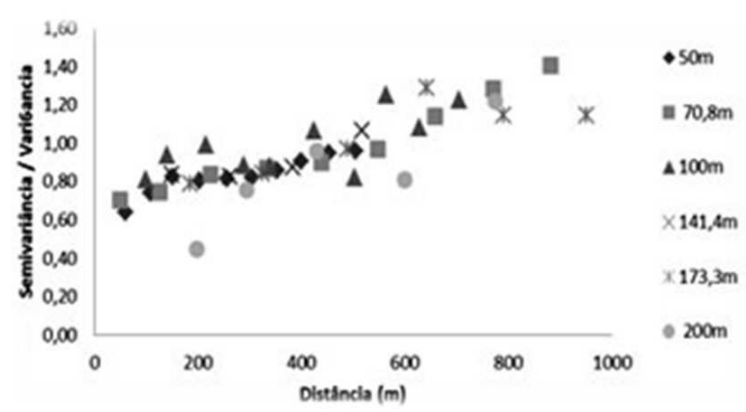

FIGURA 4. Semivariogramas de diferentes grades de amostragem para 0 atributo Carbono

A análise variográfica indicou que os atributos do solo analisados (Figura 3 e 4) separadamente nas diferentes grades regulares de amostragem apresentaram dependência espacial, com exceção do atributo: pH para grade de 141,4; 173,3 e $200 \mathrm{~m}$, que apresentaram efeito pepita puro, ou seja, com valor aproximadamente igual ao patamar $(\mathrm{C}+\mathrm{C} 0)$, no qual denomina-se efeito pepita puro, demonstrando que as amostras não recebem influência espacial (TRANGMAR et al., 1985). O atributo químico $\mathrm{pH}$ apresentou estrutura de dependência espacial fraca, com alcance (Ao) que corresponde a zona de influência da dependência espacial em torno de 240-300m nas grades de amostragem (Tabela 4). 
TABELA 4. Semivariograma dos valores de $\mathrm{pH}$ para as diferentes grades de amostragem.

\begin{tabular}{cccccc}
\hline \multicolumn{2}{c}{ Semivariograma } & Efeito Pepita & Semivâriancia & Alcance $(\mathrm{m})$ & IGF $^{1}$ \\
\hline Grade $(\mathrm{m})$ & Modelo & Co & $($ Co $+\mathrm{C})$ & A0 \\
\hline 50 & Esférico & 0,13502 & 0,16702 & $240-300$ & $9,02 \mathrm{E}-04$ \\
70,8 & Esférico & 0,1451 & 0,1971 & $270-300$ & $1,05 \mathrm{E}-02$ \\
100 & Gaussiano & 0,11502 & 0,18302 & 240 & $9,87 \mathrm{E}-03$ \\
141,4 & \multicolumn{5}{c}{ Efeito Pepita Puro } \\
173,3 & \multicolumn{5}{c}{ Efeito Pepita Puro } \\
200 & \multicolumn{5}{c}{ Efeito Pepita Puro } \\
\hline \multicolumn{5}{c}{} \\
\hline
\end{tabular}

IGF $^{1}$ (Indicação da qualidade do ajuste).

A partir da grade regular de $100 \mathrm{~m}$ este atributo não obteve dependência espacial sendo que, nas grades de 50, 70,8 e 100m o efeito pepita (Co) e a semivâriancia $(\mathrm{C} 0+\mathrm{C})$ apresentaram valores muito próximo, indicando que o conjunto de dados tem tendência espacial que se aproxima do efeito pepita puro.

Tal condição pode ser justificada pelo fato dessa área estar recebendo correções (calagem) há muito tempo, em concordância com as afirmações de CAMBARDELLA et al. (1994), de que a dependência espacial fraca ou a ocorrência de estrutura não definida se deve as variações extrínsecas, causadas pela aplicação de fertilizantes, preparo do solo e outras práticas de cultivo. BERG \& KLAMT (1997) também concluíram que a maior variância para $\mathrm{pH}$ a curtas distâncias $(50 \mathrm{~m})$ mostrouse relacionada à distribuição não homogênea de calcário.

O atributo Carbono apresentou estrutura do semivariograma com dependência espacial moderada em todas as grades regulares estabelecidas (Tabelas 5), concordando com resultados obtido por SALVIANO et al. (1998) e SOUZA et al. (1998). O Carbono apresentou um alcance em torno de $300-800 \mathrm{~m}$, com continuidade espacial maior que os demais atributos da área de estudo.

TABELA 5. Semivariograma dos valores de Carbono para as diferentes grades de amostragem.

\begin{tabular}{llllll}
\hline \multicolumn{2}{l}{ Semivariograma } & Efeito Pepita & Semivâriancia & Alcance $(\mathrm{m})$ & IGF $^{1}$ \\
\hline Grade $(\mathrm{m})$ & Modelo & Co & $($ Co $+\mathrm{C})$ & $\mathrm{A} 0$ & \\
\hline 50 & Exponencial & 19,8 & 37,3 & 510 & $4,47 \mathrm{E}-03$ \\
70,8 & Esférico & 26,7 & 40,0 & $540-630$ & $1,94 \mathrm{E}-02$ \\
100 & Esférico & 24,9 & 34,1 & $580-600$ & $1,44 \mathrm{E}-02$ \\
141,4 & Gaussiano & 21,1 & 31,8 & 800 & $1,76 \mathrm{E}-02$ \\
173,3 & Gaussiano & 26,8 & 42,3 & 780 & $1,74 \mathrm{E}-02$ \\
200 & Gaussiano & 15,2 & 61,2 & 780 & $1,62 \mathrm{E}-01$ \\
\hline \multicolumn{5}{c}{ IGF $^{1}$ (Indicação da qualidade do ajuste). }
\end{tabular}

Os semivariogramas do atributo Carbono foram ajustados em sua maioria no modelo gaussiano. Os valores de alcance e a estrutura de dependência espacial para 
Carbono podem ser atribuídos à forma de distribuição desses elementos no solo, assim como, manejo e manutenção dos restos culturais (palha) são geralmente queimados e ficam distribuídos uniformemente sobre o solo, na área de estudo, como também destacado por NANNI (2001).

Os valores do alcance relativos aos semivariogramas têm uma importância considerável na determinação do limite da dependência espacial, podendo ser também um indicativo do intervalo entre unidades de mapeamento de solos (GREGO \& VIEIRA, 2005), sendo que as classes de dependência espacial moderadas pode serem atribuídas aos fatores intrínsecos (mineralogia e textura), ao passo que aos extrínsecos (manejo do solo) atribui-se fraca dependência.

A dependência espacial moderada seria devido à homogeneização do solo, confirmado esta hipótese quando se analisa a amplitude de variação dos atributos químicos Carbono, pH que apresentam CV baixo, situação semelhante foi observada por CAVALCANTE et al. (2007) estudando sistemas de uso e manejo do solo. Mapas de distribuição espacial dos atributos químicos $\mathrm{pH}$ e Carbono foram gerados a partir da interpolação por krigagem das grades que obtiveram dependência espacial, Figuras (5) letras A,B e C são do atributo pH grades regulares de 50,70,8 e $100 \mathrm{~m}$ respectivamente, letras $\mathrm{D}, \mathrm{E}, \mathrm{F}, \mathrm{G}, \mathrm{H}$ e I são do atributo Carbono grades regulares de $50,70,8,100,141,4,173,3$ e $200 \mathrm{~m}$ respectivamente.

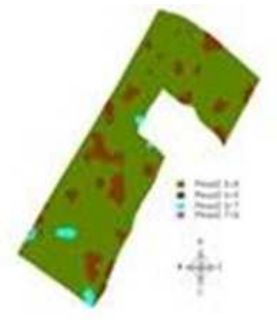

A

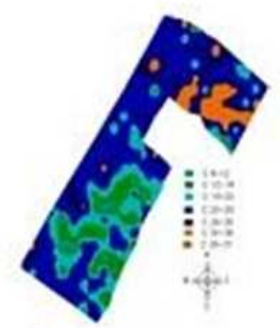

D

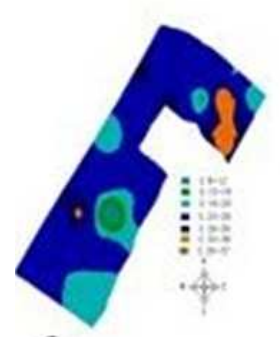

G

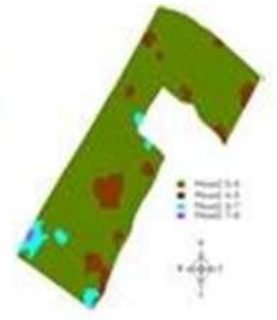

B

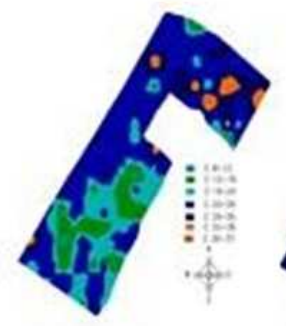

$E$

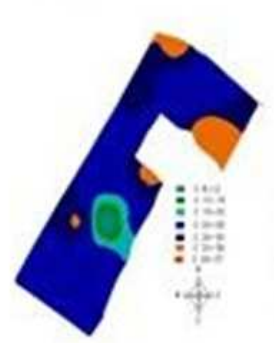

$\mathrm{H}$

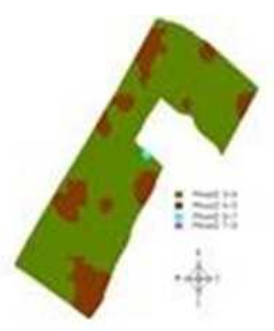

C.

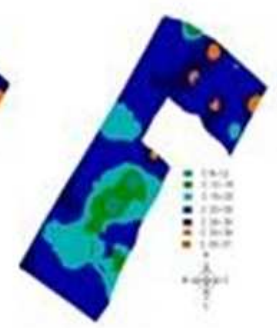

F

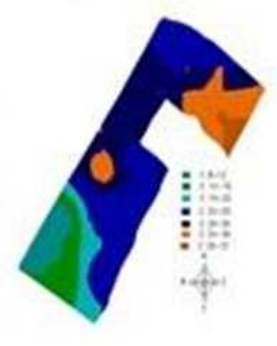

1 
FIGURA 5. Mapas de distribuição espacial dos atributos $\mathrm{pH}$ e Carbono

\section{CONCLUSÕES}

A estatística descritiva dos atributos da área de estudo analisados em conjunto com diferentes grades regulares de amostragem demonstrou homogeneidade química, ou seja, se a opção for pelas medidas de tendência central, todas as grades apresentaram resultados muito próximos, independente da intensidade de amostragem.

Nas análises semivariográfica todos os atributos apresentaram dependência espacial: pH, com exceções: pH grade de 141,4, 173,3 e 200m.

Os resultados obtidos nos semivariogramas de forma separada para cada atributo em suas grades regulares de amostragem estabeleceram qual grade regular com menor densidade de amostras o atributo pode ser caracterizado de forma satisfatório e representativo pela geoestatística: - pH grade regular de 100m (uma amostra a cada um hectare); - Carbono grade regular de 173,3m (uma amostra a cada três hectares).

\section{REFERÊNCIAS}

CAMARA, G. Conceitos Básicos em Ciência da Geoinformação. 1998. Disponível em: < http://www.dpi.inpe.br/gilberto/livro/introd/index.htm> Acesso em: dez. 2006.

CAMBARDELLA, C. A.; MOORMAN, T. B.; NOVAK, J. M.; PARKIN, T. B.; KARLEN, D. L. TURCO; R. F., KONOPKA, A. E. Field-scale variability of soil propierties in Central lowa soils. Soil Science Society of American Journal, v.58, n.5, p.1501-11, 1994.

CAVAlCANTE, E. G. S.; Alves, M. C.; SOUZA, Z. M. de; PeReIRA, G. T. Variabilidade espacial de atributos químicos do solo sob diferentes usos e manejos. Revista Brasileira de Ciência do Solo, v.31, n.6, p.1329-1339, 2007.

DAVIS, J. G.; HOSSNER, L. R.; WILDING, L. P.; MANU, A. 1995. Variability of soil chemical properties in two sandy dunal soils of niger. Soil Sci.. v. 159, p. 321-330.

EMBRAPA, Centro nacional de Pesquisa de Solos. Manual de métodos de análise de solo. 2. ed. Rio de Janeiro, 2010. 212p.

GONÇALVES, A.C.A. Variabilidade espacial de propriedades físicas do solo para fins de manejo da irrigação. Piracicaba, 1997. 118p. Tese (Doutorado) - Escola Superior de Agricultura "Luiz de Queiroz", Universidade de São Paulo.

GREGO, R.G.; VIEIRA, S.R. Variabilidade espacial de propriedades físicas do solo em uma parcela experimental. Revista Brasileira de Ciência do Solo, v.29, p.169-177, 2005. 
McBRATNEY, A. B.; WEBSTER, R. How many observations are need for regional estimation of soil properties. Soil Science, v.135, n.3, p.177-83, 1983.

MOREIRA, L.G.; VIANA, T.V.A.; AZEVEDO, B.M.; ANDRADE JUNIOR, A.S.; COSTA, S.C. Valores recomendáveis de precipitação pluvial para uso no manejo da irrigação em Russas, CE. Revista Ciência Agronômica, Fortaleza, v.34, n.2, p.241-245, 2003.

NANNI, M.R., POVH, F.P., DEMATTÊ, J.A.M., DE OLIVEIRA, R.B., CHICATI, M.L., CEZAR, E. Optimum size in grid soil sampling for variable rate application in sitespecific management. Scientia Agricola, v.68, n.3, p.386-392, 2011.

PANNATIER, Y. Variowin: software for spatial data analysis in 2D. New York: SpringerVerlag, 1996. 90p.

SALVIANO, A.A.C.; VIEIRA, S.R.; SPAROVEK, G. Variabilidade espacial de atributos de solo e de Crotalaria juncea $L$. em área severamente erodida. Revista Brasileira de Ciência do Solo v.22, n.1, p 115-122, 1998.

SILVA, A.A. Estudo de diferentes grades regulares de amostragem para avaliação de atributos químicos do solo e mapas de fertilidade em área de cultivo de cana de açucar. Maringá 2013. 5p. Dissertação de mestrado - Universidade Estadual de Maringá.

SOUZA, L.S.; COGO, N.P.; VIERA, S.R. Variabilidade de fósforo, potássio e matéria orgânica no solo, em relação a sistemas de manejo. Revista Brasileira de Ciência do Solo, v.22, n.3, p 77-86, 1998.

TRANGMAR, B. B.; YOST, R. S.; UEHARA, G. Application of geostatistics to spatial studies of soil properties. Adv. Agron., v.38, p.45-94, 1985.

VANNI, S.M. Modelos de regressão: Estatística aplicada. SãoPaulo, Legmar Informática, 1998. 177p.

VIEIRA, S. R. Variabilidade espacial de argila, silte e atributos químicos em uma parcela experimental de um latossolo roxo de Campinas (SP). Bragantia, Campinas, v. 56, n. 1, p. 1-17, 1997.

WARRICK, A.W.; NIELSEN, D.R. Spatial variability of same physical properties of the soil. In: Hill, D. ed. Aplications of soil physics, New York: Academic Press, 1980, Cap. 13, p. 319-344.

WEBSTER, R.; OLIVER, M.A. Statistical methods in soil and land resource survey. Oxford: Oxford University Press, 1990. 316p. 